\title{
VECTOR VALUED INEQUALITIES AND LITTLEWOOD-PALEY OPERATORS ON HARDY SPACES
}

\author{
SHUICHI SATO
}

\begin{abstract}
We prove certain vector valued inequalities on $\mathbb{R}^{n}$ related to LittlewoodPaley theory. They can be used in proving characterization of the Hardy spaces in terms of Littlewood-Paley operators by methods of real analysis.
\end{abstract}

\section{INTRODUCTION}

We consider the Littlewood-Paley function on $\mathbb{R}^{n}$ defined by

$$
g_{\varphi}(f)(x)=\left(\int_{0}^{\infty}\left|f * \varphi_{t}(x)\right|^{2} \frac{d t}{t}\right)^{1 / 2}
$$

where $\varphi_{t}(x)=t^{-n} \varphi\left(t^{-1} x\right)$. We assume that $\varphi \in L^{1}\left(\mathbb{R}^{n}\right)$ and

$$
\int_{\mathbb{R}^{n}} \varphi(x) d x=0 .
$$

If we further assume that $|\varphi(x)| \leq C(1+|x|)^{-n-\epsilon}$ for some $\epsilon>0$, then we have

$$
\left\|g_{\varphi}(f)\right\|_{p} \leq C_{p}\|f\|_{p}, \quad 1<p<\infty
$$

where $\|f\|_{p}=\|f\|_{L^{p}}$ (see [10] and also [1] for an earlier result). The reverse inequality also holds if a certain non-degeneracy condition on $\varphi$ is assumed in addition (see [7, Theorem 3.8] and also [11). This is the case for $g_{Q}$ with $Q(x)=$ $[(\partial / \partial t) P(x, t)]_{t=1}$, where $P(x, t)$ is the Poisson kernel associated with the upper half space $\mathbb{R}^{n} \times(0, \infty)$ defined by

$$
P(x, t)=c_{n} \frac{t}{\left(|x|^{2}+t^{2}\right)^{(n+1) / 2}}
$$

with $c_{n}=\pi^{-(n+1) / 2} \Gamma((n+1) / 2)$ (see [12, Chap. I]). Here we recall that $\hat{Q}(\xi)=$ $-2 \pi|\xi| e^{-2 \pi|\xi|}$, where the Fourier transform is defined as

$$
\hat{f}(\xi)=\mathcal{F}(f)(\xi)=\int_{\mathbb{R}^{n}} f(x) e^{-2 \pi i\langle x, \xi\rangle} d x, \quad\langle x, \xi\rangle=x_{1} \xi_{1}+\cdots+x_{n} \xi_{n} .
$$

Furthermore, it is known that

$$
c_{1}\|f\|_{H^{p}} \leq\left\|g_{Q}(f)\right\|_{p} \leq c_{2}\|f\|_{H^{p}}
$$

for $f \in H^{p}\left(\mathbb{R}^{n}\right)$ (the Hardy space), $0<p<\infty$, where $c_{1}, c_{2}$ are positive constants (see [4] and also [14). Recall that a tempered distribution $f$ belongs to $H^{p}\left(\mathbb{R}^{n}\right)$ if $\|f\|_{H^{p}}=\left\|f^{*}\right\|_{p}<\infty$, where $f^{*}(x)=\sup _{t>0}\left|\Phi_{t} * f(x)\right|$. Here $\Phi$ is in $\mathcal{S}\left(\mathbb{R}^{n}\right)$ and satisfies $\int \Phi(x) d x=1$, where $\mathcal{S}\left(\mathbb{R}^{n}\right)$ denotes the Schwartz class of rapidly

2010 Mathematics Subject Classification. Primary 42B25; Secondary $42 B 30$.

Key Words and Phrases. Vector valued inequalities, Littlewood-Paley functions, Hardy spaces.

The author is partly supported by Grant-in-Aid for Scientific Research (C) No. 25400130, Japan Society for the Promotion of Science. 
decreasing smooth functions on $\mathbb{R}^{n}$; it is known that any other choice of such $\Phi$ gives an equivalent norm (see [4]).

In this note we are concerned with the first inequality of (1.3) for $0<p \leq 1$. A proof of the inequality was given by Uchiyama [14. The proof is based on real analysis methods and does not use special properties of the Poisson kernel such as harmonicity, a semigroup property. Consequently, 14 can also prove

$$
\|f\|_{H^{p}} \leq c\left\|g_{\varphi}(f)\right\|_{p}, \quad 0<p \leq 1,
$$

for $\varphi \in \mathcal{S}\left(\mathbb{R}^{n}\right)$ satisfying (1.2) and a suitable non-degeneracy condition. Also, a relation between Hardy spaces on homogeneous groups and Littlewood-Paley functions associated with the heat kernel can be found in [5, Chap. 7].

On the other hand, it is known and would be seen by applying an easier version of our arguments in the following that the Peetre maximal function $F_{N, R}^{* *}$ can be used along with familiar methods to prove (1.4) when $\varphi \in \mathcal{S}\left(\mathbb{R}^{n}\right)$ with a non-degeneracy condition and with the condition $\operatorname{supp}(\hat{\varphi}) \subset\left\{a_{1} \leq|\xi| \leq a_{2}\right\}, a_{1}, a_{2}>0$, where for a function $F$ on $\mathbb{R}^{n}$ and positive real numbers $N, R$, the maximal function is defined as

$$
F_{N, R}^{* *}(x)=\sup _{y \in \mathbb{R}^{n}} \frac{|F(x-y)|}{(1+R|y|)^{N}}
$$

(see [8]).

The purpose of this note is to prove (1.4) for a class of functions $\varphi$ including $Q$ and a general $\varphi \in \mathcal{S}\left(\mathbb{R}^{n}\right)$, without the restriction on $\operatorname{supp}(\hat{\varphi})$ above, with (1.2) and an admissible non-degeneracy condition (Corollary 3.2) as an application of a vector valued inequality which will be shown by using the maximal function $F_{N, R}^{* *}$ (see Proposition 2.3. Theorem 2.10 below). The proof of Proposition 2.3 consists partly in further developing methods of $[13$, Chap. V] and it admits some weighted inequalities. Theorem 2.10 follows from Proposition 2.3. Our proofs of Proposition 2.3 and Corollary 3.2 are fairly straightforward and they will be expected to extend to some other situations.

In Section 2, Proposition 2.3 will be formulated in a general form, while Theorem 2.10 will be stated in a more convenient form for the application to the proof of Corollary 3.2. In Section 3, we shall apply Theorem 2.10 and an atomic decomposition for Hardy spaces to prove Corollary 3.2. Finally, in Section 4, we shall give proofs of Lemmas 2.1 and 2.5] in Section 2 from [13] and [8, respectively, for completeness; the lemmas will be needed in proving Proposition 2.3

\section{VECTOR VALUED INEQUALITIES}

Let $\varphi^{(j)}, j=1,2, \ldots, M$, be functions in $L^{1}\left(\mathbb{R}^{n}\right)$ satisfying the non-degeneracy condition

$$
\inf _{\xi \in \mathbb{R}^{n} \backslash\{0\}} \sup _{t>0} \sum_{j=1}^{M}\left|\mathcal{F}\left(\varphi^{(j)}\right)(t \xi)\right|>c
$$

for some positive constant $c$. We write $\varphi=\left(\varphi^{(1)}, \ldots, \varphi^{(M)}\right), \hat{\varphi}=\left(\mathcal{F}\left(\varphi^{(1)}\right), \ldots, \mathcal{F}\left(\varphi^{(M)}\right)\right)$.

Lemma 2.1. Let $\varphi^{(j)}, j=1,2, \ldots, M$, be functions in $L^{1}\left(\mathbb{R}^{n}\right)$ satisfying (2.1). Then, there exist $b_{0} \in(0,1)$ and positive numbers $r_{1}, r_{2}$ with $r_{1}<r_{2}$ such that if $b \in\left[b_{0}, 1\right)$, we can find $\eta=\left(\eta^{(1)}, \ldots, \eta^{(M)}\right)$ which satisfies the following:

(1) $\eta \in C^{\infty}\left(\mathbb{R}^{n}\right)$, where $\eta \in C^{k}(U)$ means $\eta^{(j)} \in C^{k}(U)$ for all $1 \leq j \leq M$; 
(2) $\operatorname{supp} \mathcal{F}\left(\eta^{(j)}\right) \subset\left\{r_{1}<|\xi|<r_{2}\right\}, 1 \leq j \leq M$;

(3) each $\mathcal{F}\left(\eta^{(j)}\right)$ is continuous, $1 \leq j \leq M$;

(4) $\sum_{j=-\infty}^{\infty}\left\langle\hat{\varphi}\left(b^{j} \xi\right), \hat{\eta}\left(b^{j} \xi\right)\right\rangle=1 \quad$ for $\xi \in \mathbb{R}^{n} \backslash\{0\}$, where $\langle z, w\rangle=\sum_{j=1}^{M} z_{j} w_{j}$, $z, w \in \mathbb{C}^{M}$ (the Cartesian product of $M$ copies of the set of complex numbers).

Further, if $\hat{\varphi} \in C^{k}\left(\mathbb{R}^{n} \backslash\{0\}\right)$, then $\hat{\eta} \in C^{k}\left(\mathbb{R}^{n}\right)$.

See [13, Chap. V] and also [2].

We assume that $M=1$ for simplicity. Suppose that $\psi \in L^{1}\left(\mathbb{R}^{n}\right)$ and there exist $\Theta \in C^{\infty}\left(\mathbb{R}^{n}\right)$ and $A \geq 1$ such that

$$
\hat{\psi}(\xi)=\hat{\varphi}(\xi) \Theta(\xi) \quad \text { on }\left\{|\xi|<r_{2} A^{-1}\right\} .
$$

Suppose that $b \in\left[b_{0}, 1\right)$ and let $\eta$ be as in Lemma 2.1] with $M=1$. For $J>0$, define $\zeta_{J}$ by

$$
\hat{\zeta}_{J}(\xi)=1-\sum_{j: b^{j} \leq J} \hat{\varphi}\left(b^{j} \xi\right) \hat{\eta}\left(b^{j} \xi\right) .
$$

We note that $\operatorname{supp}\left(\hat{\zeta}_{J}\right) \subset\left\{|\xi| \leq r_{2} J^{-1}\right\}, \hat{\zeta}_{J}=1$ in $\left\{|\xi|<r_{1} J^{-1}\right\}$. By (2.2) it follows that

$$
\begin{aligned}
\hat{\psi}(\xi) & =\sum_{j: b^{j} \leq A} \hat{\psi}(\xi) \hat{\varphi}\left(b^{j} \xi\right) \hat{\eta}\left(b^{j} \xi\right)+\hat{\zeta}_{A}(\xi) \hat{\psi}(\xi) \\
& =\sum_{j: b^{j} \leq A} \hat{\varphi}\left(b^{j} \xi\right) \mathcal{F}\left(\alpha^{\left(b^{j}\right)}\right)\left(b^{j} \xi\right)+\hat{\varphi}(\xi) \hat{\beta}(\xi),
\end{aligned}
$$

where $\alpha^{\left(b^{j}\right)}(x)=\psi_{b^{-j}} * \eta(x)$ and $\hat{\beta}(\xi)=\hat{\zeta}_{A}(\xi) \Theta(\xi)$.

Let $E(\psi, f)(x, t)=f * \psi_{t}(x), f \in \mathcal{S}\left(\mathbb{R}^{n}\right)$. Then we have

$$
|E(\psi, f)(x, t)| \leq \sum_{j: b^{j} \leq A}\left|E\left(\alpha^{\left(b^{j}\right)} * \varphi, f\right)\left(x, b^{j} t\right)\right|+|E(\beta * \varphi, f)(x, t)| .
$$

Also, let $E_{\psi}(x, t)=E(\psi, f)(x, t)$, when $f$ is fixed.

Define

$$
C_{0}(\psi, t, L, x)=(1+|x|)^{L}\left|\int \hat{\psi}\left(t^{-1} \xi\right) \hat{\eta}(\xi) e^{2 \pi i\langle x, \xi\rangle} d \xi\right|, \quad t>0, L \geq 0 .
$$

Consequently,

$$
\left|\alpha_{s}^{\left(b^{j}\right)}(x)\right|=C_{0}\left(\psi, b^{j}, L, x / s\right) s^{-n}(1+|x| / s)^{-L}
$$

for $j \in \mathbb{Z}$ (the set of integers). Likewise, we have

$$
\left|\beta_{s}(x)\right|=D(\Theta, A, L, x / s) s^{-n}(1+|x| / s)^{-L},
$$

where

$$
D(\Theta, J, L, x)=(1+|x|)^{L}\left|\int \hat{\zeta}_{J}(\xi) \Theta(\xi) e^{2 \pi i\langle x, \xi\rangle} d \xi\right| .
$$

Here $\hat{\zeta}_{J}$ is as in (2.3). We also write $C(\psi, j, L, x)=C_{0}\left(\psi, b^{j}, L, x\right), j \in \mathbb{Z}$. Let

$$
\begin{gathered}
C(\psi, j, L)=\int_{\mathbb{R}^{n}} C(\psi, j, L, x) d x, \quad j \in \mathbb{Z}, \\
D(\Theta, J, L)=\int_{\mathbb{R}^{n}} D(\Theta, J, L, x) d x .
\end{gathered}
$$


We also write $C(\psi, j, L)=C_{\varphi}(\psi, j, L), D(\Theta, J, L)=D_{\varphi}(\Theta, J, L)$ to indicate that these quantities are based on $\varphi$. See Lemma 2.8 below for a sufficient condition which implies $C(\psi, j, L)<\infty, D(\Theta, J, L)<\infty$.

The maximal function in (1.5) is used in the following result.

Lemma 2.2. Let $\varphi, \psi \in L^{1}\left(\mathbb{R}^{n}\right)$. Suppose that $\varphi$ satisfies (2.1). Let $b \in\left[b_{0}, 1\right)$. We assume that $\psi$ and $\varphi$ are related by (2.2) with $\Theta \in C^{\infty}\left(\mathbb{R}^{n}\right)$ and $A \geq 1$. Let $N>0$. Then for $f \in \mathcal{S}\left(\mathbb{R}^{n}\right)$, we have

$$
\begin{aligned}
&|E(\psi, f)(x, t)| \leq C \sum_{j: b^{j} \leq A} C(\psi, j, N) E(\varphi, f)\left(\cdot, b^{j} t\right)_{N,\left(b^{j} t\right)^{-1}}^{* *}(x) \\
&+C D(\Theta, A, N) E(\varphi, f)(\cdot, t)_{N, t^{-1}}^{* *}(x) \\
& E(\psi, f)(\cdot, t)_{N, t^{-1}}^{* *}(x) \leq C \sum_{j: b^{j} \leq A} C(\psi, j, N) b^{-j N} E(\varphi, f)\left(\cdot, b^{j} t\right)_{N,\left(b^{j} t\right)^{-1}}^{* *}(x) \\
&+C D(\Theta, A, N) E(\varphi, f)(\cdot, t)_{N, t^{-1}}^{* *}(x) .
\end{aligned}
$$

Proof. Using (2.4), we see that

$$
\begin{aligned}
& \left|E_{\psi}(z, t)\right| \\
& \leq C \sum_{j: b^{j} \leq A} \int\left|E_{\varphi}\left(y, b^{j} t\right)\right|\left(1+\frac{|z-y|}{b^{j} t}\right)^{-N} C\left(\psi, j, N,(z-y) /\left(b^{j} t\right)\right)\left(b^{j} t\right)^{-n} d y \\
& +C \int\left|E_{\varphi}(y, t)\right|\left(1+\frac{|z-y|}{t}\right)^{-N} D(\Theta, A, N,(z-y) / t) t^{-n} d y .
\end{aligned}
$$

If we multiply both sides of the inequality by $(1+|x-z| / t)^{-N}$ and observe that

$$
\left(1+\frac{|z-y|}{b^{j} t}\right)^{-N}\left(1+\frac{|x-z|}{t}\right)^{-N} \leq C_{A, N} b^{-N j}\left(1+\frac{|x-y|}{b^{j} t}\right)^{-N}
$$

for all $x, y, z \in \mathbb{R}^{n}$ and $t>0$ under the condition $b^{j} \leq A$, then we see that

$$
\begin{aligned}
& \left|E_{\psi}(z, t)\right|(1+|x-z| / t)^{-N} \\
& \leq C \sum_{j: b^{j} \leq A} b^{-N j} \int\left|E_{\varphi}\left(y, b^{j} t\right)\right|\left(1+\frac{|x-y|}{b^{j} t}\right)^{-N} C\left(\psi, j, N,(z-y) /\left(b^{j} t\right)\right)\left(b^{j} t\right)^{-n} d y \\
& \quad+C \int\left|E_{\varphi}(y, t)\right|\left(1+\frac{|x-y|}{t}\right)^{-N} D(\Theta, A, N,(z-y) / t) t^{-n} d y,
\end{aligned}
$$

and hence

$$
\begin{aligned}
& \left|E_{\psi}(z, t)\right|(1+|x-z| / t)^{-N} \\
& \leq C \sum_{j: b^{j} \leq A} b^{-N j} E_{\varphi}\left(\cdot, b^{j} t\right)_{N,\left(b^{j} t\right)^{-1}}^{* *}(x) \int C\left(\psi, j, N,(z-y) /\left(b^{j} t\right)\right)\left(b^{j} t\right)^{-n} d y \\
& \quad+C E_{\varphi}(\cdot, t)_{N, t^{-1}}^{* *}(x) \int D(\Theta, A, N,(z-y) / t) t^{-n} d y \\
& \leq C \sum_{j: b^{j} \leq A} C(\psi, j, N) b^{-N j} E_{\varphi}\left(\cdot, b^{j} t\right)_{N,\left(b^{j} t\right)^{-1}}^{* *}(x)+C D(\Theta, A, N) E_{\varphi}(\cdot, t)_{N, t^{-1}}^{* *}(x) .
\end{aligned}
$$


The estimate (2.10) follows by taking the supremum in $z$ over $\mathbb{R}^{n}$. The proof of (2.9) is easier; putting $z=x$ and arguing as above, we get (2.9).

Let $\varphi \in L^{1}\left(\mathbb{R}^{n}\right)$. Suppose that $\varphi$ satisfies (2.1). Let $L>0$. We consider the following conditions.

$$
\begin{gathered}
\varphi \in C^{1}\left(\mathbb{R}^{n}\right), \quad \partial_{k} \varphi \in L^{1}\left(\mathbb{R}^{n}\right), \quad 1 \leq k \leq n \\
|\hat{\varphi}(\xi)| \leq C|\xi|^{\epsilon} \quad \text { for some } \epsilon>0 ; \\
\sup _{j \geq 0} C_{\varphi}(\nabla \varphi, j, L) b^{-j L-\epsilon j}<\infty \quad \text { for some } \epsilon>0, \text { together with (2.11); } \\
D_{\varphi}(L)<\infty, \quad \text { with (2.11), }
\end{gathered}
$$

where we write $\nabla \varphi=\left(\partial_{1} \varphi, \ldots, \partial_{n} \varphi\right), \partial_{k}=\partial_{x_{k}}=\partial / \partial_{x_{k}}$ and $C_{\varphi}(\nabla \varphi, j, L)=$ $\sum_{k=1}^{n} C_{\varphi}\left(\partial_{k} \varphi, j, L\right)$; also we define $D_{\varphi}(L)=\sum_{k=1}^{n} D_{\varphi}\left(\Xi_{k}, 1, L\right)$ by taking $\Theta(\xi)=$ $\Xi_{k}(\xi)=2 \pi i \xi_{k}$ and $J=1$ in (2.8). We note that (2.11) implies the following (with $\epsilon=1)$ :

$$
|\hat{\varphi}(\xi)| \leq C|\xi|^{-\epsilon} \quad \text { for some } \epsilon>0 .
$$

Let $\psi \in L^{1}\left(\mathbb{R}^{n}\right)$. We assume that $\psi$ is related to $\varphi$ as in (2.2) with $\Theta \in C^{\infty}\left(\mathbb{R}^{n}\right)$ and $A \geq 1$. We also consider the conditions:

$$
\begin{gathered}
\sup _{j: b^{j} \leq A} C_{\varphi}(\psi, j, L) b^{-\epsilon j}<\infty \text { for some } \epsilon>0 \\
D_{\varphi}(\Theta, A, L)<\infty .
\end{gathered}
$$

Let $M$ be the Hardy-Littlewood maximal operator

$$
M(f)(x)=\sup _{x \in B}|B|^{-1} \int_{B}|f(y)| d y,
$$

where the supremum is taken over all balls $B$ in $\mathbb{R}^{n}$ such that $x \in B$ and $|B|$ denotes the Lebesgue measure of $B$. Let $1<p<\infty$. We recall that a weight function $w$ belongs to the weight class $A_{p}$ of Muckenhoupt on $\mathbb{R}^{n}$ if

$$
[w]_{A_{p}}=\sup _{B}\left(|B|^{-1} \int_{B} w(x) d x\right)\left(|B|^{-1} \int_{B} w(x)^{-1 /(p-1)} d x\right)^{p-1}<\infty,
$$

where the supremum is taken over all balls $B$ in $\mathbb{R}^{n}$ Also, we recall that a weight function $w$ is in the class $A_{1}$ if $M(w) \leq C w$ almost everywhere. The infimum of all such $C$ is denoted by $[w]_{A_{1}}$.

For a weight $w$, the weighted $L^{p}$ norm is defined as

$$
\|f\|_{p, w}=\left(\int_{\mathbb{R}^{n}}|f(x)|^{p} w(x) d x\right)^{1 / p}
$$

We have the following vector value inequality.

Proposition 2.3. Let $\varphi \in L^{1}\left(\mathbb{R}^{n}\right)$. We assume that $\varphi$ satisfies (2.1) with $M=1$. Let $N>0, n / N<p, q<\infty$ and $w \in A_{p N / n}$. Suppose that $\varphi$ satisfies (2.11), (2.12) and (2.13), (2.14) with $L=N$. Let $\psi \in L^{1}\left(\mathbb{R}^{n}\right)$. Suppose that $\psi$ is related to $\varphi$ as in (2.2) with $\Theta \in C^{\infty}\left(\mathbb{R}^{n}\right), A \geq 1$ and (2.16), (2.17) hold with $L=N$. Then

$$
\left\|\left(\int_{0}^{\infty}\left|f * \psi_{t}\right|^{q} \frac{d t}{t}\right)^{1 / q}\right\|_{p, w} \leq C\left\|\left(\int_{0}^{\infty}\left|f * \varphi_{t}\right|^{q} \frac{d t}{t}\right)^{1 / q}\right\|_{p, w}
$$

for $f \in \mathcal{S}\left(\mathbb{R}^{n}\right)$ with a positive constant $C$ independent of $f$. 
We need the next result to show Proposition 2.3.

Lemma 2.4. Suppose that $0<q<\infty, N>0$ and that $\varphi \in L^{1}\left(\mathbb{R}^{n}\right)$ satisfies (2.1), (2.11), (2.12) and (2.13), (2.14) with $L=N$. Then

$$
\int_{0}^{\infty} E(\varphi, f)(\cdot, t)_{N, t^{-1}}^{* *}(x)^{q} \frac{d t}{t} \leq C \int_{0}^{\infty} M\left(\left|f * \varphi_{t}\right|^{r}\right)(x)^{q / r} \frac{d t}{t}, \quad r=n / N .
$$

We need the following in proving Lemma 2.4

Lemma 2.5 (see [8]). If $F \in C^{1}\left(\mathbb{R}^{n}\right)$ and $R>0, r>0$, then

$$
F_{N, R}^{* *}(x) \leq C \delta^{-N} M\left(|F|^{r}\right)(x)^{1 / r}+C \delta R^{-1}|\nabla F|_{N, R}^{* *}(x)
$$

for all $\delta \in(0,1]$, where $N=n / r$ and the constant $C$ is independent of $\delta$ and $R$.

Proof of Lemma 2.4. By Lemma 2.5 we have

$$
E(\varphi, f)(\cdot, t)_{N, t^{-1}}^{* *}(x) \leq C \delta^{-N} M\left(\left|f * \varphi_{t}\right|^{r}\right)(x)^{1 / r}+C \delta\left|f *(\nabla \varphi)_{t}\right|_{N, t^{-1}}^{* *}(x),
$$

where $f *(\nabla \varphi)_{t}=\left(f *\left(\partial_{1} \varphi\right)_{t}, \ldots, f *\left(\partial_{n} \varphi\right)_{t}\right), r=n / N$. We apply (2.10) of Lemma 2.2 with $\psi=\partial_{k} \varphi, \Theta(\xi)=2 \pi i \xi_{k}, A=1$ in (2.2). Then

$$
\begin{aligned}
& \left|f *(\nabla \varphi)_{t}\right|_{N, t^{-1}}^{* *}(x) \\
\leq & C \sum_{j \geq 0} C_{\varphi}(\nabla \varphi, j, N) b^{-j N} E(\varphi, f)\left(\cdot, b^{j} t\right)_{N,\left(b^{j} t\right)^{-1}}^{* *}(x)+C D_{\varphi}(N) E(\varphi, f)(\cdot, t)_{N, t^{-1}}^{* *}(x) .
\end{aligned}
$$

Using this in (2.18) and applying Hölder's inequality when $q>1$, we see that

$$
\begin{aligned}
& E(\varphi, f)(\cdot, t)_{N, t^{-1}}^{* *}(x)^{q} \leq C \delta^{-N q} M\left(\left|f * \varphi_{t}\right|^{r}\right)(x)^{q / r} \\
& +C_{q} \delta^{q} \sum_{j \geq 0} C_{\varphi}(\nabla \varphi, j, N)^{q} b^{-j N q} b^{-\tau c_{q} j} E(\varphi, f)\left(\cdot, b^{j} t\right)_{N,\left(b^{j} t\right)^{-1}}^{* *}(x)^{q} \\
& \quad+C \delta^{q} D_{\varphi}(N)^{q} E(\varphi, f)(\cdot, t)_{N, t^{-1}}^{* *}(x)^{q} .
\end{aligned}
$$

where $\tau>0, c_{q}=1$ if $q>1$ and $c_{q}=0$ if $0<q \leq 1$.

If we integrate both sides of the inequality (2.19) over $(0, \infty)$ with respect to the measure $d t / t$ and if we apply termwise integration on the right hand side, then we have

$$
\begin{aligned}
& (2.20) \quad \int_{0}^{\infty} E(\varphi, f)(\cdot, t)_{N, t^{-1}}^{* *}(x)^{q} \frac{d t}{t} \leq C \delta^{-N q} \int_{0}^{\infty} M\left(\left|f * \varphi_{t}\right|^{r}\right)(x)^{q / r} \frac{d t}{t} \\
& +C_{q} \delta^{q}\left[\sum_{j \geq 0} C_{\varphi}(\nabla \varphi, j, N)^{q} b^{-j N q} b^{-\tau c_{q} j}+D_{\varphi}(N)^{q}\right] \int_{0}^{\infty} E(\varphi, f)(\cdot, t)_{N, t^{-1}}^{* *}(x)^{q} \frac{d t}{t} .
\end{aligned}
$$

The condition (2.13) with $L=N$ implies that the sum in $j$ on the right hand side of (2.20) is finite if $\tau$ is small enough. We can see that the last integral on the right hand side of (2.20) is finite for $f \in \mathcal{S}\left(\mathbb{R}^{n}\right)$ by (2.12) and (2.15). Further, we have (2.14) for $L=N$. Altogether, it follows that the second term on the right hand side of (2.20) is finite. Thus, we can get the conclusion if we choose $\delta$ sufficiently small. 
Proof of Proposition 2.3, By (2.9) we have

$$
\begin{aligned}
|E(\psi, f)(x, t)|^{q} \leq C_{q} \sum_{j: b^{j} \leq A} C(\psi, j, N)^{q} b^{-\tau c_{q} j} E(\varphi, f)\left(\cdot, b^{j} t\right)_{N,\left(b^{j} t\right)^{-1}}^{* *}(x)^{q} \\
+C D(\Theta, A, N)^{q} E(\varphi, f)(\cdot, t)_{N, t^{-1}}^{* *}(x)^{q},
\end{aligned}
$$

where $\tau>0$ and $c_{q}$ is as in (2.19). Integrating with the measure $d t / t$ over $(0, \infty)$, we have

$$
\begin{aligned}
& \quad \int_{0}^{\infty}|E(\psi, f)(x, t)|^{q} \frac{d t}{t} \\
& \leq C_{q}\left[\sum_{j: b^{j} \leq A} C(\psi, j, N)^{q} b^{-\tau c_{q} j}+D(\Theta, A, N)^{q}\right] \int_{0}^{\infty} E(\varphi, f)(\cdot, t)_{N, t^{-1}}^{* *}(x)^{q} \frac{d t}{t} .
\end{aligned}
$$

The sum in $j$ on the right hand side of (2.21) is finite by (2.16) with $L=N$ if $\tau$ is small enough; also we have assumed $D(\Theta, A, N)<\infty($ (2.17) with $L=N)$. Let $r=n / N<q, p$ and $w \in A_{p N / n}$. By (2.21) and Lemma 2.4 we see that

$$
\begin{aligned}
&\left(\int _ { \mathbb { R } ^ { n } } \left(\int_{0}^{\infty}\right.\right.\left.\left.|E(\psi, f)(x, t)|^{q} \frac{d t}{t}\right)^{p / q} w(x) d x\right)^{1 / p} \\
& \leq C\left\|\left(\int_{0}^{\infty} M\left(\left|f * \varphi_{t}\right|^{r}\right)(x)^{q / r} \frac{d t}{t}\right)^{1 / q}\right\|_{p, w} \\
&=C\left\|\left(\int_{0}^{\infty} M\left(\left|f * \varphi_{t}\right|^{r}\right)(x)^{q / r} \frac{d t}{t}\right)^{r / q}\right\|_{p / r, w}^{1 / r} \\
& \leq C\left(\int_{\mathbb{R}^{n}}\left(\int_{0}^{\infty}|E(\varphi, f)(x, t)|^{q} \frac{d t}{t}\right)^{p / q} w(x) d x\right)^{1 / p},
\end{aligned}
$$

where the last inequality follows form the following lemma, which is a version of the vector valued inequality for the Hardy-Littlewood maximal functions of FeffermanStein [3] (see [9] for a proof of the $\ell^{\mu}$-valued case, which may be available also in the present situation).

Lemma 2.6. Suppose that $1<\mu, \nu<\infty$ and $w \in A_{\nu}$. Then for appropriate functions $E(x, t)$ on $\mathbb{R}^{n} \times(0, \infty)$ we have

$$
\left\|\left(\int_{0}^{\infty} M\left(E^{t}\right)(x)^{\mu} \frac{d t}{t}\right)^{1 / \mu}\right\|_{\nu, w} \leq C\left(\int_{\mathbb{R}^{n}}\left(\int_{0}^{\infty}|E(x, t)|^{\mu} \frac{d t}{t}\right)^{\nu / \mu} w(x) d x\right)^{1 / \nu},
$$

where $E^{t}(x)=E(x, t)$.

This completes the proof of Proposition 2.3 ,

We have an analogous result for general $\varphi=\left(\varphi^{(1)}, \ldots, \varphi^{(M)}\right)$, although Proposition 2.3 is stated only for the case $M=1$.

It is obvious that $Q, \hat{Q}(\xi)=-2 \pi|\xi| e^{-2 \pi|\xi|}$, satisfies all the requirements on $\varphi$ in Lemma 2.4 for all $N>0$. To state results with more directly verifiable assumptions on $\varphi$ and $\psi$, we introduce a class of functions. 
Definition 2.7. Let $\psi \in L^{1}\left(\mathbb{R}^{n}\right)$. Let $l$ be a non-negative integer and $\tau$ a nonnegative real number. We say $\psi \in B_{\tau}^{l}$ if $\hat{\psi} \in C^{l}\left(\mathbb{R}^{n} \backslash\{0\}\right)$ and

$$
\left|\partial_{\xi}^{\gamma} \hat{\psi}(\xi)\right| \leq C_{\gamma}|\xi|^{-\tau-|\gamma|} \quad \text { outside a neighborhood of the origin }
$$

for every $\gamma$ satisfying $|\gamma| \leq l$ with a constant $C_{\gamma}$, where $\gamma=\left(\gamma_{1}, \ldots, \gamma_{n}\right)$ is a multi-index, $\gamma_{j} \in \mathbb{Z}, \gamma_{j} \geq 0,|\gamma|=\gamma_{1}+\cdots+\gamma_{n}$ and $\partial_{\xi}^{\gamma}=\partial_{\xi_{1}}^{\gamma_{1}} \ldots \partial_{\xi_{n}}^{\gamma_{n}}$.

Clearly, $Q \in B_{\tau}^{l}$ for any $l, \tau$. This is also the case for $\psi \in \mathcal{S}\left(\mathbb{R}^{n}\right)$.

Lemma 2.8. Suppose that $\varphi \in L^{1}\left(\mathbb{R}^{n}\right)$ and $\varphi$ satisfies the condition (2.1). Let $\tau \geq 0, J>0$ and let $L$ be a non-negative integer.

(1) Suppose that $\psi \in B_{\tau}^{L+[n / 2]+1}$ and $\hat{\varphi} \in C^{L+[n / 2]+1}\left(\mathbb{R}^{n} \backslash\{0\}\right)$, where $[a]$ denotes the largest integer not exceeding a. Then we have

$$
\sup _{j: b^{j} \leq J} C_{\varphi}(\psi, j, L) b^{-j \tau}<\infty
$$

where $C_{\varphi}(\psi, j, L)=C(\psi, j, L)$ is as in (2.7).

(2) Suppose that $\Theta \in C^{\infty}\left(\mathbb{R}^{n}\right)$ and $\hat{\varphi} \in C^{L+[n / 2]+1}\left(\mathbb{R}^{n} \backslash\{0\}\right)$. Then

$$
D_{\varphi}(\Theta, J, L)<\infty,
$$

where $D_{\varphi}(\Theta, J, L)=D(\Theta, J, L)$ is as in (2.8).

(3) Let $\psi^{(k)} \in L^{1}\left(\mathbb{R}^{n}\right)$ and $\mathcal{F}\left(\psi^{(k)}\right)(\xi)=2 \pi i \xi_{k} \hat{\varphi}(\xi), 1 \leq k \leq n$. If $\varphi \in$ $B_{L+1+\tau}^{L+[n / 2]+1}$, then we have

$$
\sup _{j: b^{j} \leq J} C_{\varphi}\left(\psi^{(k)}, j, L\right) b^{-j L-j \tau}<\infty, \quad D_{\varphi}\left(\Xi_{k}, 1, L\right)<\infty
$$

for each $k$, where $\Xi_{k}(\xi)=2 \pi i \xi_{k}$ as above.

Proof. Part (3) follows from part (1) and part (2) since $\psi^{(k)} \in B_{L+\tau}^{L+[n / 2]+1}$ and $\hat{\varphi} \in C^{L+[n / 2]+1}\left(\mathbb{R}^{n} \backslash\{0\}\right)$ if $\varphi \in B_{L+1+\tau}^{L+[n / 2]+1}$. To prove part (1), we note that

$$
\begin{aligned}
& (1+|x|)^{[n / 2]+1} C_{0}(\psi, t, L, x) \\
\leq & C\left|\int \hat{\psi}\left(t^{-1} \xi\right) \hat{\eta}(\xi) e^{2 \pi i\langle x, \xi\rangle} d \xi\right|+C \sup _{|\gamma|=L+[n / 2]+1}\left|\int \partial_{\xi}^{\gamma}\left[\hat{\psi}\left(t^{-1} \xi\right) \hat{\eta}(\xi)\right] e^{2 \pi i\langle x, \xi\rangle} d \xi\right|,
\end{aligned}
$$

where $C_{0}(\psi, t, L, x)$ is as in (2.5). We note that $\hat{\eta} \in C^{L+[n / 2]+1}\left(\mathbb{R}^{n}\right)$ by Lemma 2.1. since $\hat{\varphi} \in C^{L+[n / 2]+1}\left(\mathbb{R}^{n} \backslash\{0\}\right)$. The assumption $\psi \in B_{\tau}^{L+[n / 2]+1}$ implies

$$
\left|\partial_{\xi}^{\gamma}\left[\hat{\psi}\left(t^{-1} \xi\right) \hat{\eta}(\xi)\right]\right| \leq C_{M} t^{\tau}, \quad 0<t \leq M
$$

for any $M>0$, if $|\gamma|=L+[n / 2]+1$ or $\gamma=0$. It follows that

$$
C_{0}(\psi, t, L, x) \leq C(1+|x|)^{-[n / 2]-1} G(x)
$$

with some $G \in L^{2}$ such that $\|G\|_{2} \leq C t^{\tau}$. Thus, since $[n / 2]+1>n / 2$, by the Schwarz inequality we have

$$
\int_{\mathbb{R}^{n}} C_{0}(\psi, t, L, x) d x \leq C t^{\tau} .
$$

The conclusion of part (1) follows from (2.23) with $t=b^{j}$.

Likewise, we have

$$
\int_{\mathbb{R}^{n}} D(\Theta, J, L, x) d x<\infty
$$


under the assumptions of part (2), where $D(\Theta, J, L, x)$ is as in (2.6), which proves part (2).

By Lemma 2.8 and Proposition 2.3 we have the following.

Theorem 2.9. Let $\varphi \in L^{1}\left(\mathbb{R}^{n}\right)$ satisfy (2.1) with $M=1$. Suppose that $\psi \in L^{1}\left(\mathbb{R}^{n}\right)$ and $\hat{\psi}(\xi)=\hat{\varphi}(\xi) \Theta(\xi)$ in a neighborhood of the origin with some $\Theta \in C^{\infty}\left(\mathbb{R}^{n}\right)$. Let $0<p, q<\infty$ and let $N$ be a positive integer such that $N>\max (n / p, n / q)$. Let $w \in A_{p N / n}$. Suppose that $\varphi$ belongs to $B_{N+1+\epsilon}^{N+[n / 2]+1}$ for some $\epsilon>0$ and satisfies (2.11) and (2.12). Also, suppose that $\psi \in B_{\epsilon}^{N+[n / 2]+1}$ for some $\epsilon>0$. Then we have

$$
\left\|\left(\int_{0}^{\infty}\left|f * \psi_{t}\right|^{q} \frac{d t}{t}\right)^{1 / q}\right\|_{p, w} \leq C\left\|\left(\int_{0}^{\infty}\left|f * \varphi_{t}\right|^{q} \frac{d t}{t}\right)^{1 / q}\right\|_{p, w}
$$

for $f \in \mathcal{S}\left(\mathbb{R}^{n}\right)$, where $C$ is a positive constant independent of $f$.

Proof. If we have (2.11) and if $\varphi \in B_{N+1+\epsilon}^{N+[n / 2]+1}$, then (2.13) and (2.14) hold with $L=N$ by part (3) of Lemma 2.8 with $J=1, \tau=\epsilon, L=N$. Since $\psi \in B_{\epsilon}^{N+[n / 2]+1}$ and $\varphi \in C^{N+[n / 2]+1}\left(\mathbb{R}^{n} \backslash\{0\}\right)$, if $\hat{\psi}(\xi)=\hat{\varphi}(\xi) \Theta(\xi)$ on $\left\{|\xi|<r_{2} A^{-1}\right\}, A \geq 1$, we have (2.16) and (2.17) with $L=N$ by part (1) of Lemma 2.8 with $J=A$, $\tau=\epsilon, L=N$ and part (2) of Lemma 2.8 with $J=A, L=N$, respectively. Thus Proposition 2.3 implies the conclusion.

This immediately implies the following.

Theorem 2.10. Let $\varphi \in L^{1}\left(\mathbb{R}^{n}\right)$ satisfy (2.1) with $M=1$, (2.11) and (2.12). We assume that $0<p, q<\infty$ and $N$ is a positive integer satisfying $N>\max (n / p, n / q)$. Let $w \in A_{p N / n}$. Suppose that $\varphi \in B_{N+1+\epsilon}^{N+[n / 2]+1}$ for some $\epsilon>0$. Then, if $\psi \in \mathcal{S}\left(\mathbb{R}^{n}\right)$ and $\hat{\psi}$ vanishes in a neighborhood of the origin, the inequality

$$
\left\|\left(\int_{0}^{\infty}\left|f * \psi_{t}\right|^{q} \frac{d t}{t}\right)^{1 / q}\right\|_{p, w} \leq C\left\|\left(\int_{0}^{\infty}\left|f * \varphi_{t}\right|^{q} \frac{d t}{t}\right)^{1 / q}\right\|_{p, w}, \quad f \in \mathcal{S}\left(\mathbb{R}^{n}\right),
$$

holds with a positive constant $C$ independent of $f$.

Proof. We see that $\hat{\psi}(\xi)=\hat{\varphi}(\xi) \Theta(\xi)$ in a neighborhood of the origin with $\Theta$ being identically 0 . Obviously, $\psi \in B_{1}^{N+[n / 2]+1}$. So all the requirements for $\varphi$ and $\psi$ in Theorem 2.9] are satisfied. Thus the conclusion follows from Theorem 2.9, This completes the proof.

We note that $Q$ fulfills all the requirements on $\varphi$ in Theorem 2.10 for every $N$. The same is true of $\varphi_{0} \in \mathcal{S}\left(\mathbb{R}^{n}\right)$ satisfying (2.1) (with $M=1$ ) and (1.2).

\section{Littlewood-Paley operators AND Hardy SPACES}

Let $\mathcal{H}$ denote the Hilbert space of functions $u(t)$ on $(0, \infty)$ such that $\|u\|_{\mathcal{H}}=$ $\left(\int_{0}^{\infty}|u(t)|^{2} d t / t\right)^{1 / 2}<\infty$. We first recall Hardy spaces of functions on $\mathbb{R}^{n}$ with values in $\mathcal{H}$, which will be used to prove (1.4) by Theorem 2.10 (see Corollary 3.2 below).

The Lebesgue space $L_{\mathcal{H}}^{q}\left(\mathbb{R}^{n}\right)$ consists of functions $h(y, t)$ with the norm

$$
\|h\|_{q, \mathcal{H}}=\left(\int_{\mathbb{R}^{n}}\left\|h^{y}\right\|_{\mathcal{H}}^{q} d y\right)^{1 / q}
$$


where $h^{y}(t)=h(y, t)$. For $0<p \leq 1$, we consider the Hardy space $H_{\mathcal{H}}^{p}\left(\mathbb{R}^{n}\right)$ of functions on $\mathbb{R}^{n}$ with values in $\mathcal{H}$. We take $\varphi \in \mathcal{S}\left(\mathbb{R}^{n}\right)$ with $\int \varphi(x) d x=1$. Let $h \in L_{\mathcal{H}}^{2}\left(\mathbb{R}^{n}\right)$. We recall that $h \in H_{\mathcal{H}}^{p}\left(\mathbb{R}^{n}\right)$ if $\|h\|_{H_{\mathcal{H}}^{p}}=\left\|h^{*}\right\|_{L^{p}}<\infty$, where

$$
h^{*}(x)=\sup _{s>0}\left(\int_{0}^{\infty}\left|\varphi_{s} * h^{t}(x)\right|^{2} \frac{d t}{t}\right)^{1 / 2}
$$

with $h^{t}(x)=h(x, t)$.

If $a$ is a $(p, \infty)$ atom in $H_{\mathcal{H}}^{p}\left(\mathbb{R}^{n}\right)$, we have

(i) $\left(\int_{0}^{\infty}|a(x, t)|^{2} d t / t\right)^{1 / 2} \leq|Q|^{-1 / p}$, where $Q$ is a cube in $\mathbb{R}^{n}$ with sides parallel to the coordinate axes;

(ii) $\sup (a(\cdot, t)) \subset Q$ uniformly in $t>0$, where $Q$ is the same as in $(i)$;

(iii) $\int_{\mathbb{R}^{n}} a(x, t) x^{\gamma} d x=0$ for all $t>0$ and $\gamma$ such that $|\gamma| \leq[n(1 / p-1)]$, where $\gamma=\left(\gamma_{1}, \ldots, \gamma_{n}\right)$ is a multi-index and $x^{\gamma}=x_{1}^{\gamma_{1}} \ldots x_{n}^{\gamma_{n}}$.

We apply the following atomic decomposition.

Lemma 3.1. Let $h \in L_{\mathcal{H}}^{2}\left(\mathbb{R}^{n}\right)$. If $h \in H_{\mathcal{H}}^{p}\left(\mathbb{R}^{n}\right)$, then there exist a sequence $\left\{a_{k}\right\}$ of $(p, \infty)$ atoms in $H_{\mathcal{H}}^{p}\left(\mathbb{R}^{n}\right)$ and a sequence $\left\{\lambda_{k}\right\}$ of positive numbers such that $\sum_{k=1}^{\infty} \lambda_{k}^{p} \leq C\|h\|_{H_{\mathcal{H}}^{p}}^{p}$ with a constant $C$ independent of $h$ and $h=\sum_{k=1}^{\infty} \lambda_{k} a_{k}$ in $H_{\mathcal{H}}^{p}\left(\mathbb{R}^{n}\right)$ and in $L_{\mathcal{H}}^{2}\left(\mathbb{R}^{n}\right)$.

A proof of the atomic decomposition for $H^{p}\left(\mathbb{R}^{n}\right)$ can be found in [6] and [13. Similar methods apply to the vector valued case.

In this section, we prove the following result as an application of Theorem 2.10 .

Corollary 3.2. Let $0<p \leq 1, N>n / p$. Suppose that $\varphi \in L^{1}\left(\mathbb{R}^{n}\right)$ satisfies (2.1) with $M=1$, (2.11), (2.12) and suppose that $\varphi \in B_{N+1+\epsilon}^{N+[n / 2]+1}$ for some $\epsilon>0$. Then we have

$$
\|f\|_{H^{p}} \leq C_{p}\left\|g_{\varphi}(f)\right\|_{p}
$$

for $f \in H^{p}\left(\mathbb{R}^{n}\right) \cap \mathcal{S}\left(\mathbb{R}^{n}\right)$, where $C_{p}$ is a positive constant independent of $f$.

This can be generalized to an arbitrary $f \in H^{p}\left(\mathbb{R}^{n}\right)$ if $\varphi=Q$ or if $\varphi$ is a function in $\mathcal{S}\left(\mathbb{R}^{n}\right)$ satisfying (2.1) and (1.2) (see 14).

In proving Corollary 3.2 , we need the following.

Lemma 3.3. Suppose that $\eta \in \mathcal{S}\left(\mathbb{R}^{n}\right), \operatorname{supp}(\hat{\eta}) \subset\{1 / 2 \leq|\xi| \leq 4\}, \hat{\eta}(\xi)=1$ on $\{1 \leq|\xi| \leq 2\}$ and that $\Phi \in \mathcal{S}\left(\mathbb{R}^{n}\right)$ satisfies $\int_{\mathbb{R}^{n}} \Phi(x) d x=1$. Let $\psi \in \mathcal{S}\left(\mathbb{R}^{n}\right)$ and $\operatorname{supp} \hat{\psi} \subset\{1 \leq|\xi| \leq 2\}$. Then, for $p, q>0$ and $f \in \mathcal{S}\left(\mathbb{R}^{n}\right)$ we have

$$
\left\|\left(\int_{0}^{\infty} \sup _{s>0}\left|\Phi_{s} * \psi_{t} * f\right|^{q} \frac{d t}{t}\right)^{1 / q}\right\|_{p} \leq C\left\|\left(\int_{0}^{\infty}\left|\eta_{t} * f\right|^{q} \frac{d t}{t}\right)^{1 / q}\right\|_{p} .
$$

Proof. We note that $\hat{\Phi}(s \xi) \hat{\psi}(t \xi)=\hat{\Phi}(s \xi) \hat{\psi}(t \xi) \hat{\eta}(t \xi)$. Thus we have

$$
\begin{aligned}
\left|\Phi_{s} * \psi_{t} * f(x)\right| & \leq\left(f * \eta_{t}\right)_{N, t^{-1}}^{* *}(x) \int_{\mathbb{R}^{n}}\left|\Phi_{s} * \psi_{t}(w)\right|\left(1+t^{-1}|w|\right)^{N} d w \\
& =\left(f * \eta_{t}\right)_{N, t^{-1}}^{* *}(x) \int_{\mathbb{R}^{n}}\left|\Phi_{s / t} * \psi(w)\right|(1+|w|)^{N} d w \\
& \leq C_{N}\left(f * \eta_{t}\right)_{N, t^{-1}}^{* *}(x)
\end{aligned}
$$

for any $N>0$, with a positive constant $C_{N}$ independent of $s, t$. The last inequality follows from the observation that $\Phi_{s / t} * \psi, s, t>0$, belongs to a bounded subset 
of the topological vector space $\mathcal{S}\left(\mathbb{R}^{n}\right)$, since $\mathcal{F}\left(\Phi_{u} * \psi\right)(\xi)=\hat{\Phi}(u \xi) \hat{\psi}(\xi), u>0$, and $\hat{\psi}(\xi)$ is supported on $\{1 \leq|\xi| \leq 2\}$. Therefore, we have

$$
\left(\int_{0}^{\infty} \sup _{s>0}\left|\Phi_{s} * \psi_{t} * f(x)\right|^{q} \frac{d t}{t}\right)^{1 / q} \leq C\left(\int_{0}^{\infty}\left|\left(f * \eta_{t}\right)_{N, t^{-1}}^{* *}(x)\right|^{q} \frac{d t}{t}\right)^{1 / q} .
$$

Thus (3.1) and Lemma 2.4 with $\eta$ in place of $\varphi$ imply

$$
\left(\int_{0}^{\infty} \sup _{s>0}\left|\Phi_{s} * \psi_{t} * f(x)\right|^{q} \frac{d t}{t}\right)^{1 / q} \leq C\left(\int_{0}^{\infty} M\left(\left|f * \eta_{t}\right|^{r}\right)(x)(x)^{q / r} \frac{d t}{t}\right)^{1 / q},
$$

with $N=n / r$. By this and Lemma 2.6 the conclusion follows as in (2.22).

We also use the following to prove Corollary 3.2

Lemma 3.4. Let $\hat{\psi} \in \mathcal{S}\left(\mathbb{R}^{n}\right)$ be a radial function supported on $\{1 \leq|\xi| \leq 2\}$ such that

$$
\int_{0}^{\infty}|\hat{\psi}(t \xi)|^{2} \frac{d t}{t}=1 \quad \text { for all } \xi \neq 0 .
$$

Let $f \in H^{p}\left(\mathbb{R}^{n}\right) \cap \mathcal{S}\left(\mathbb{R}^{n}\right), 0<p \leq 1$, and put $E(y, t)=f * \psi_{t}(y)$. Then $E$ is in $H_{\mathcal{H}}^{p}\left(\mathbb{R}^{n}\right)$ and we have

$$
\|f\|_{H^{p}} \leq C\|E\|_{H_{\mathcal{H}}^{p}} .
$$

Let $\psi$ be a function in $L^{1}\left(\mathbb{R}^{n}\right)$ satisfying (1.2). Suppose that $h \in L_{\mathfrak{H}}^{2}$. Let $h_{(\epsilon)}(y, t)=h(y, t) \chi_{\left(\epsilon, \epsilon^{-1}\right)}(t), 0<\epsilon<1$, where $\chi_{S}$ denotes the characteristic function of a set $S$. Put

$$
F_{\psi}^{\epsilon}(h)(x)=\int_{0}^{\infty} \int_{\mathbb{R}^{n}} \psi_{t}(x-y) h_{(\epsilon)}(y, t) d y \frac{d t}{t} .
$$

To prove Lemma 3.4 we apply the following.

Lemma 3.5. Let $0<p \leq 1$. Suppose that $\psi \in \mathcal{S}\left(\mathbb{R}^{n}\right)$ and $\operatorname{supp} \hat{\psi} \subset\{1 \leq|\xi| \leq 2\}$. Then

$$
\sup _{\epsilon \in(0,1)}\left\|F_{\psi}^{\epsilon}(h)\right\|_{H^{p}} \leq C\|h\|_{H_{\mathcal{H}}^{p}}
$$

Proof. Let $a$ be a $(p, \infty)$ atom in $H_{\mathcal{H}}^{p}\left(\mathbb{R}^{n}\right)$ with support in the cube $Q$ of the definition of the atom. We denote by $y_{0}$ the center of $Q$. Let $\widetilde{Q}$ be a concentric enlargement of $Q$ such that $2\left|y-y_{0}\right|<\left|x-y_{0}\right|$ if $y \in Q$ and $x \in \mathbb{R}^{n} \backslash \widetilde{Q}$. Let $\Phi$ be a non-negative $C^{\infty}$ function on $\mathbb{R}^{n}$ supported on $\{|x|<1\}$ which satisfies $\int \Phi(x) d x=1$. Let $\Psi_{s, t}=\Phi_{s} * \psi_{t}, s, t>0$. Then $\Psi_{s, t}=\left(\Phi_{s / t} * \psi\right)_{t}$ and $\Phi_{u} * \psi$, $u>0$, belongs to a bounded subset of the topological vector space $\mathcal{S}\left(\mathbb{R}^{n}\right)$, as in the proof of Lemma 3.3

Let $P_{x}\left(y, y_{0}\right)$ be the Taylor polynomial in $y$ of order $M=[n(1 / p-1)]$ at $y_{0}$ for $\Phi_{s / t} * \psi(x-y)$. Then, if $\left|x-y_{0}\right|>2\left|y-y_{0}\right|$, we see that

$$
\left|\Phi_{s / t} * \psi(x-y)-P_{x}\left(y, y_{0}\right)\right| \leq C\left|y-y_{0}\right|^{M+1}\left(1+\left|x-y_{0}\right|\right)^{-L}
$$

where $L>n+M+1$ and the constant $C$ is independent of $s, t, x, y, y_{0}$, and hence

$$
\left|\Psi_{s, t}(x-y)-t^{-n} P_{x / t}\left(y / t, y_{0} / t\right)\right| \leq C t^{-n-M-1}\left|y-y_{0}\right|^{M+1}\left(1+\left|x-y_{0}\right| / t\right)^{-L} .
$$


SHUICHI SATO

Therefore, by the properties of an atom and the Schwarz inequality, for $x \in \mathbb{R}^{n} \backslash \widetilde{Q}$ we have

$$
\begin{aligned}
& \left|\Phi_{s} * F_{\psi}^{\epsilon}(a)(x)\right|=\left|\iint_{\mathbb{R}^{n} \times(0, \infty)}\left(\Psi_{s, t}(x-y)-t^{-n} P_{x / t}\left(y / t, y_{0} / t\right)\right) a_{(\epsilon)}(y, t) d y \frac{d t}{t}\right| \\
& \leq \int_{Q}\left(\int_{0}^{\infty}\left|\Psi_{s, t}(x-y)-t^{-n} P_{x / t}\left(y / t, y_{0} / t\right)\right|^{2} \frac{d t}{t}\right)^{1 / 2}\left(\int_{0}^{\infty}|a(y, t)|^{2} \frac{d t}{t}\right)^{1 / 2} d y \\
& \leq C|Q|^{-1 / p} \int_{Q}\left(\int_{0}^{\infty}\left|\Psi_{s, t}(x-y)-t^{-n} P_{x / t}\left(y / t, y_{0} / t\right)\right|^{2} \frac{d t}{t}\right)^{1 / 2} d y \\
& \leq C|Q|^{-1 / p} \int_{Q}\left|y-y_{0}\right|^{M+1}\left|x-y_{0}\right|^{-n-M-1} d y \\
& \leq C|Q|^{-1 / p+1+(M+1) / n}\left|x-y_{0}\right|^{-n-M-1} .
\end{aligned}
$$

We note that $p>n /(n+M+1)$. Thus

$$
\begin{aligned}
& \int_{\mathbb{R}^{n} \backslash \widetilde{Q}} \sup _{s>0}\left|\Phi_{s} * F_{\psi}^{\epsilon}(a)(x)\right|^{p} d x \\
& \leq C|Q|^{-1+p+p(M+1) / n} \int_{\mathbb{R}^{n} \backslash \widetilde{Q}}\left|x-y_{0}\right|^{-p(n+M+1)} \leq C .
\end{aligned}
$$

Since $\int_{0}^{\infty}|\hat{\psi}(t \xi)|^{2} d t / t \leq C$, by duality we have

$$
\sup _{\epsilon \in(0,1)}\left\|F_{\psi}^{\epsilon}(h)\right\|_{2} \leq C\|h\|_{L_{\mathcal{H}}^{2}}, \quad h \in L_{\mathcal{H}}^{2}\left(\mathbb{R}^{n}\right) .
$$

Thus, applying Hölder's inequality, by the properties (i), (ii) of $a$ we see that

$$
\begin{aligned}
\int_{\widetilde{Q}} \sup _{s>0}\left|\Phi_{s} * F_{\psi}^{\epsilon}(a)(x)\right|^{p} d x & \leq C|Q|^{1-p / 2}\left(\int_{\widetilde{Q}}\left|M\left(F_{\psi}^{\epsilon}(a)\right)(x)\right|^{2} d x\right)^{p / 2} \\
& \leq C|Q|^{1-p / 2}\left(\int_{Q} \int_{0}^{\infty}|a(y, t)|^{2} \frac{d t}{t} d y\right)^{p / 2} \\
& \leq C .
\end{aligned}
$$

The estimates (3.2) and (3.3) imply

$$
\int_{\mathbb{R}^{n}} \sup _{s>0}\left|\Phi_{s} * F_{\psi}^{\epsilon}(a)(x)\right|^{p} d x \leq C .
$$

Using Lemma 3.1 and (3.4), we have

$$
\int_{\mathbb{R}^{n}} \sup _{s>0}\left|\Phi_{s} * F_{\psi}^{\epsilon}(h)(x)\right|^{p} d x \leq C\|h\|_{H_{\mathcal{H}}^{p}}^{p}
$$

This completes the proof.

Proof of Lemma 3.4. The fact that $E \in H_{\mathcal{H}}^{p}\left(\mathbb{R}^{n}\right)$ can be proved similarly to the proof of Lemma [3.5] by applying the atomic decomposition for $H^{p}\left(\mathbb{R}^{n}\right.$ ) (see [14, Lemma 3.6]).

We write

$$
F_{\widetilde{\psi}}^{\epsilon}(E)(x)=\int_{\epsilon}^{\epsilon^{-1}} \int_{\mathbb{R}^{n}} \psi_{t} * f(y) \bar{\psi}_{t}(y-x) d y \frac{d t}{t}=\int_{\mathbb{R}^{n}} \Psi^{(\epsilon)}(x-z) f(z) d z,
$$


where $\bar{\psi}$ denotes the complex conjugate, $\widetilde{g}(x)=g(-x)$ and

$$
\Psi^{(\epsilon)}(x)=\int_{\epsilon}^{\epsilon^{-1}} \int_{\mathbb{R}^{n}} \psi_{t}(x+y) \bar{\psi}_{t}(y) d y \frac{d t}{t} .
$$

We note that

$$
\widehat{\Psi^{(\epsilon)}}(\xi)=\int_{\epsilon}^{\epsilon^{-1}} \hat{\psi}(t \xi) \widehat{\bar{\psi}}(-t \xi) \frac{d t}{t}=\int_{\epsilon}^{\epsilon^{-1}}|\hat{\psi}(t \xi)|^{2} \frac{d t}{t} .
$$

From this and Lemma 3.5 we have

$$
\|f\|_{H^{p}} \leq C \liminf _{\epsilon \rightarrow 0}\left\|F_{\widetilde{\psi}}^{\epsilon}(E)\right\|_{H^{p}} \leq C\|E\|_{H_{\mathcal{H}}^{p}} .
$$

Proof of Corollary 3.2. We take a function $\eta$ as in Lemma 3.3. Then by Lemma 3.3 with $q=2$ and Lemma 3.4, it follows that

$$
\|f\|_{H^{p}} \leq C\left\|g_{\eta}(f)\right\|_{p}
$$

for $f \in H^{p}\left(\mathbb{R}^{n}\right) \cap \mathcal{S}\left(\mathbb{R}^{n}\right)$. If we use this and Theorem 2.10 with $q=2, w=1$ and with $\eta$ in place of $\psi$, we can reach the conclusion of Corollary 3.2 .

We can also prove discrete parameter versions of Proposition 2.3 and Corollary 3.2 by analogous methods.

Proposition 3.6. Let $N>0, n / N<p, q<\infty$. Suppose that $w \in A_{p N / n}$ and that $\varphi$ and $\psi$ fulfill the hypotheses of Proposition 2.3 with $N$. Then, for $f \in \mathcal{S}\left(\mathbb{R}^{n}\right)$ we have

$$
\left\|\left(\sum_{j=-\infty}^{\infty}\left|f * \psi_{b^{j}}\right|^{q}\right)^{1 / q}\right\|_{p, w} \leq C\left\|\left(\sum_{j=-\infty}^{\infty}\left|f * \varphi_{b^{j}}\right|^{q}\right)^{1 / q}\right\|_{p, w} .
$$

Corollary 3.7. Let $0<p \leq 1$ and $N>n / p$. Suppose that $\varphi$ fulfills the hypotheses of Corollary 3.2 with $N$. Then, for $f \in H^{p}\left(\mathbb{R}^{n}\right) \cap \mathcal{S}\left(\mathbb{R}^{n}\right)$ we have

$$
\|f\|_{H^{p}} \leq C\left\|\left(\sum_{j=-\infty}^{\infty}\left|f * \varphi_{b^{j}}\right|^{2}\right)^{1 / 2}\right\|_{p} .
$$

Also, from Proposition 3.6 we have discrete parameter analogues of Theorems 2.9 and 2.10.

\section{Proofs of Lemmas 2.1 and 2.5}

In this section we give proofs of Lemmas 2.1 and 2.5 for completeness.

Proof of Lemma 2.1. There exist a finite family $\left\{I_{j}\right\}_{j=1}^{L}$ of compact intervals in $(0, \infty)$ and a positive constant $c$ such that

$$
\inf _{\xi \in S^{n-1}} \max _{1 \leq j \leq L} \inf _{t \in I_{j}} \sum_{i=1}^{M}\left|\mathcal{F}\left(\varphi^{(i)}\right)(t \xi)\right|^{2} \geq c
$$

where $S^{n-1}=\{\xi:|\xi|=1\}$. This follows from a compactness argument, since each $\mathcal{F}\left(\varphi^{(j)}\right)$ is continuous. 
SHUICHI SATO

Let $b_{0}=\max _{1 \leq h \leq L}\left(a_{h} / b_{h}\right)$, where $I_{h}=\left[a_{h}, b_{h}\right]$. Then $b_{0} \in(0,1)$ and if $b \in$ $\left[b_{0}, 1\right), t>0$ and $1 \leq h \leq L, h \in \mathbb{Z}$, we have $b^{j} t \in I_{h}$ for some $j \in \mathbb{Z}$.

Let $[m, H]$ be an interval in $(0, \infty)$ such that $\cup_{j=1}^{L} I_{j} \subset[m, H]$. We take a nonnegative function $\theta \in C_{0}^{\infty}(\mathbb{R})$ such that $\theta=1$ on $[m, H]$, $\operatorname{supp} \theta \subset[m / 2,2 H]$. Then

$$
\sum_{j=-\infty}^{\infty} \theta\left(b^{j}|\xi|\right) \sum_{i=1}^{M}\left|\mathcal{F}\left(\varphi^{(i)}\right)\left(b^{j} \xi\right)\right|^{2}=: \Psi(\xi) \geq c>0 \quad \text { for } \xi \neq 0 .
$$

We have $\Psi\left(b^{k} \xi\right)=\Psi(\xi)$ for $k \in \mathbb{Z}$. Define

$$
\mathcal{F}\left(\eta^{(j)}\right)(\xi)=\theta(|\xi|) \overline{\mathcal{F}\left(\varphi^{(j)}\right)(\xi)} \Psi(\xi)^{-1} \quad \text { for } \xi \neq 0
$$

and $\mathcal{F}\left(\eta^{(j)}\right)(0)=0$. Then, $\eta$ has all the properties required in the lemma. Also, from the construction, we can see that $\hat{\eta} \in C^{k}\left(\mathbb{R}^{n}\right)$ if $\hat{\varphi} \in C^{k}\left(\mathbb{R}^{n} \backslash\{0\}\right)$. This completes the proof.

Proof of Lemma 2.5. Let $f_{B(x, t)} f(y) d y=|B(x, t)|^{-1} \int_{B(x, t)} f(y) d y$, where $B(x, t)$ denotes a ball in $\mathbb{R}^{n}$ with center $x$ and radius $t$. Then, for $u, r>0$ and $x, z \in \mathbb{R}^{n}$,

$$
\begin{aligned}
|F(x-z)| & =\left(f_{B(x-z, u)}|F(y)+(F(x-z)-F(y))|^{r} d y\right)^{1 / r} \\
& \leq C_{r}\left(f_{B(x-z, u)}|F(y)|^{r} d y\right)^{1 / r}+C_{r}\left(f_{B(x-z, u)}|F(x-z)-F(y)|^{r} d y\right)^{1 / r},
\end{aligned}
$$

where $C_{r}=1$ if $r \geq 1$ and $C_{r}=2^{-1+1 / r}$ if $0<r<1$. Therefore

$$
|F(x-z)| \leq C_{r}\left(f_{B(x-z, u)}|F(y)|^{r} d y\right)^{1 / r}+C_{r} \sup _{y:|x-z-y|<u} u|\nabla F(y)| .
$$

If $|x-z-y|<u,|x-y|<u+|z|$. Thus we have

$$
\begin{aligned}
|\nabla F(y)| & \leq \frac{|\nabla F(x+(y-x))|}{(1+R|x-y|)^{N}}(1+R(u+|z|))^{N} \\
& \leq|\nabla F|_{N, R}^{* *}(x)(1+\delta+R|z|)^{N} \\
& \leq 2^{N}|\nabla F|_{N, R}^{* *}(x)(1+R|z|)^{N}
\end{aligned}
$$

if we choose $u=\delta / R$. Consequently,

$$
\sup _{y:|x-z-y|<u} u|\nabla F(y)| \leq 2^{N} u|\nabla F|_{N, R}^{* *}(x)(1+R|z|)^{N}
$$

with $u=\delta / R$.

Also, if $u=\delta / R$,

$$
\begin{aligned}
& \left(f_{B(x-z, u)}|F(y)|^{r} d y\right)^{1 / r} \leq\left(u^{-n}(u+|z|)^{n} f_{B(x, u+|z|)}|F(y)|^{r} d y\right)^{1 / r} \\
& \leq u^{-n / r}(u+|z|)^{n / r} M\left(|F|^{r}\right)(x)^{1 / r} \\
& =\delta^{-n / r}(\delta+R|z|)^{n / r} M\left(|F|^{r}\right)(x)^{1 / r} \\
& \leq \delta^{-n / r}(1+R|z|)^{n / r} M\left(|F|^{r}\right)(x)^{1 / r} .
\end{aligned}
$$


From (4.1), (4.2) and (4.3), we see that

$|F(x-z)| \leq C_{r} \delta^{-n / r}(1+R|z|)^{n / r} M\left(|F|^{r}\right)(x)^{1 / r}+2^{N} C_{r} u|\nabla F|_{N, R}^{* *}(x)(1+R|z|)^{N}$.

If $N=n / r$, it follows that

$$
\frac{|F(x-z)|}{(1+R|z|)^{N}} \leq C_{r} \delta^{-N} M\left(|F|^{r}\right)(x)^{1 / r}+2^{N} C_{r} \delta R^{-1}|\nabla F|_{N, R}^{* *}(x) .
$$

Thus we have the conclusion of the lemma by taking the supremum in $z$ over $\mathbb{R}^{n}$.

\section{REFERENCES}

[1] A. Benedek, A. P. Calderón and R. Panzone, Convolution operators on Banach space valued functions, Proc. Nat. Acad. Sci. U. S. A. 48 (1962), 356-365.

[2] A. P. Calderón and A. Torchinsky, Parabolic maximal functions associated with a distribution, Advances in Math. 16 (1975), 1-64.

[3] C. Fefferman and E. M. Stein, Some maximal inequalities, Amer. J. Math. 93 (1971), 107115.

[4] C. Fefferman and E. M. Stein, $H^{p}$ spaces of several variables, Acta Math. 129 (1972), 137193.

[5] G. B. Folland and E. M. Stein, Hardy Spaces on Homogeneous Groups, Princeton Univ. Press, Princeton, N.J. 1982.

[6] J. Garcia-Cuerva and J.L. Rubio de Francia, Weighted norm inequalities and related topics, North-Holland, Amsterdam, New York, Oxford, 1985.

[7] L. Hörmander, Estimates for translation invariant operators in $L^{p}$ spaces, Acta Math. 104 (1960), 93-139.

[8] J. Peetre, On spaces of Triebel-Lizorkin type, Ark. Mat. 13 (1975), 123-130.

[9] J. L. Rubio de Francia, F. J. Ruiz and J. L. Torrea, Calderón-Zygmund theory for operatorvalued kernels, Adv. in Math. 62 (1986), 7-48.

[10] S. Sato, Remarks on square functions in the Littlewood-Paley theory, Bull. Austral. Math. Soc. 58 (1998), 199-211.

[11] S. Sato, Littlewood-Paley equivalence and homogeneous Fourier multipliers, preprint (2015).

[12] E. M. Stein and G. Weiss, Fourier Analysis on Euclidean Spaces, Princeton Univ. Press, 1971.

[13] J. -O. Strömberg and A. Torchinsky, Weighted Hardy spaces, Lecture Notes in Math. 1381, Springer-Verlag, Berlin Heidelberg New York London Paris Tokyo Hong Kong, 1989.

[14] A. Uchiyama, Characterization of $H^{p}\left(\mathbb{R}^{n}\right)$ in terms of generalized Littlewood-Paley $g$ functions, Studia Math. 81 (1985), 135-158.

Department of Mathematics, Faculty of Education, Kanazawa University, Kanazawa 920-1192, JAPAN

E-mail address: shuichi@kenroku.kanazawa-u.ac.jp 